\title{
Les organisations paysannes : émergence et devenirs
}

Daniel Thiéba

\section{OpenEdition}

12 Journals

Édition électronique

URL : http://journals.openedition.org/apad/382

DOI : 10.4000/apad.382

ISSN : 1950-6929

Éditeur

LIT Verlag

Édition imprimée

Date de publication : 15 mars 1992

\section{Référence électronique}

Daniel Thiéba, «Les organisations paysannes : émergence et devenirs », Bulletin de l'APAD [En ligne], 3 | 1992, mis en ligne le 06 juillet 2006, consulté le 08 septembre 2020. URL : http:// journals.openedition.org/apad/382 ; DOI : https://doi.org/10.4000/apad.382

Ce document a été généré automatiquement le 8 septembre 2020

Bulletin de l'APAD 


\title{
Les organisations paysannes : émergence et devenirs
}

\author{
Daniel Thiéba
}

Mécanismes d'émergence

1 Que ce soit sous la forme de classes d'âge, de regroupement sur la base de l'appartenance sexuelle, religieuse ou encore lignagère, les associations avaient un rôle important dans les activités économiques, politiques et culturelles de l'Afrique.

2 Ainsi en est-il chez les Serer du Bawol au Sénégal pour lesquels nous présentons les formes associatives ayant précédé les organisations paysannes actuelles. Le mal (appellation serer) est la première forme. Dirigé par un maad, l'association regroupe des personnes à l'échelle du village; les activités concernent essentiellement les champ collectifs et des travaux d'intérêt commun au village. Au mal succéda le blok, dont l'origine de l'appellation reste à élucider, puis l'amicale, puis enfin les associations contemporaines désignées sous le nom de Groupements villageois.

3 Les formes précédant les Groupements se caractérisent ainsi : 1) les produits des activités économiques sont consommés lors de fêtes réunissant les membres;2) les ressources proviennent des seules activités de l'association; 3) les contacts avec les institutions de développement sont rarissimes sinon inexistants.

4 Ces associations vont connaître des évolutions diverses en fonction des contraintes et opportunités du milieu, ainsi que de la stratégie des acteurs locaux. Depuis deux décennies, on assiste à l'émergence de nouvelles formes associatives dénommées groupement villageois. Ils correspondent à des réponses spécifiques à la crise alimentaire et aux années de sécheresse (1973-1975 puis 1980 et 1983). A partir du début des années 1970, les emplois urbains commencent à se raréfier pour les migrants. Dans le même temps, l'aide alimentaire et les subventions, sous forme de dons ou crédits se dirigent vers les villages sous la houlette de la coopération bilatérale et surtout de nouveaux acteurs de la coopération internationale: les ONG. Tout en présentant de nombreuses variantes et des référents souvent fort différents, les ONG œuvrent dans le sens de "l'auto-promotion". Ceci signifie pour les initiateurs du 
concept que l'aide, matérialisée sous forme de projets, doit se greffer sur une volonté locale matérialisée sous forme associative.

5 En Afrique de l'Ouest, l'ONG Six S (Se nourrir de la Savane en Saison Sèche au Sahel) va partir de Ouayigouya, au Burkina, et de Genève, en Suisse, pour organiser une stratégie d'appui à des groupements villageois dans les pays du Sahel. Ces nouveaux cadres associatifs permettent de drainer des sommes importantes en vue de nouvelles activités économiques pendant la saison sèche (maraîchage, embauche...). Des subventions autorisent des investissements dans les villages où existent les Groupements.

6 L'essor des groupements et leur mise en fédération, à l'échelle régionale ou même nationale, va être l'œuvre de leaders presque tous revenus au village mais ayant travaillé au sein de l'administration, et bien au fait de l'idéologie d'auto-promotion chère aux partenaires étrangers. Pour ces leaders, les nouveaux cadres associatifs sont le moyen de constitution d'un pouvoir aussi bien économique que politique.

7 Dans les pays où existait une ouverture politique tolérant des organisations non dépendantes du parti unique, l'État ne s'opposera pas à la constitution des organisations paysannes. Très tôt une fraction des agents de l'État y verra la possibilité de drainer des financements extérieurs et de permettre de se désengager de certaines charges. Le discours actuel sur l'auto-promotion participe de la volonté étatique de transférer aux producteurs des activités non rentables. C'est la convergence des intérêts et perceptions de ces quatre catégories d'acteurs (coopération, paysans, nouveaux leaders, État) qui explique la croissance rapide de ce nouveau type d'association au Sahel et plus particulièrement au Sénégal et au Burkina Faso.

D. Rahmaton (Organisations Paysannes en Afrique: Problèmes et perspectives, CODESRIA, Document de travail 1,1991) définit l'organisation paysanne comme une "structure formelle ou informelle à laquelle prennent part paysans et paysannes et dont l'objectif majeur est la poursuite d'avantages communs qu'ils obtiennent contre des obligations communes". On peut avancer quelques critères permettant de distinguer ce que nous appelons organisations paysannes des anciennes formes d'association. Celles qui font l'objet de notre intérêt aujourd'hui présentent les caractéristiques suivantes : 1) elles mènent des activités économiques durant la contre-saison ; 2) elles ont des relations avec des acteurs du développement situés hors du village (services étatiques, coopération internationale...); 3) elles affichent une volonté de rechercher des solutions et alternatives à la crise agricole actuelle en restant au village ; 4) elles ont une "autonomie", tant du point de vue des activités à mener que des règles de fonctionnement.

Une grande diversité

9 Sous ces caractéristiques générales, on retrouve une grande variété d'organisations paysannes :

- certaines sont des transcroissances d'anciennes formes associatives (avec des normes de fonctionnement culturel ancrées), tandis que d'autres empruntent largement aux référents externes d'organisation ;

- certaines sont exclusives, d'autres non. Dans le premier cas le critère d'exclusion pourrait être : le sexe, l'âge, le lignage d'appartenance, la religion. L'exclusion est rarement inscrite formellement, mais le plus souvent il s'agit de règles implicites dans le trame des stratégies ;

- certaines sont fédérées à des entités régionales, voire nationales, alors que d'autres se limitent à un village ; 
- certaines disposent d'un budget se chiffrant à des dizaines de millions de francs, réalisent des investissements à caractère collectif et entretiennent d'étroites relations avec une bonne brochette de partenaires extérieurs. D'autres ont un budget modeste constitué en grande partie des cotisations. pratiques et des discours divers liés à la multiplicité des caractéristiques énoncées ci-dessus.

Enjeux, devenirs

11 Les sciences sociales ont aussi un champ de recherche à couvrir pour dégager les conditions de pérennité des associations paysannes et de leurs transcroissance en mouvement social. Non pas à travers un discours normatif, mais par une analyse dégageant les significations et implications des choix opérés par les acteurs. Il ne s'agit pas "de prévoir des événements, mais d'analyser les mécanismes par lesquels se forment l'action collective et, au niveau le plus élevé, les mouvements sociaux". (A. Touraine : Le retour de l'acteur, Fayard : 209).

12 Les associations paysannes en Afrique noire sont aujourd'hui à la croisée des chemins. Après deux décennies de parcours, on peut esquisser un regard rétrospectif et interrogateur.

13 1- Face à la déconfiture des organisations coopératives créées par les institutions de l'État, les associations paysannes se représentent comme de nouveaux lieux tenant un discours et des pratiques autour du thème Développement. Elles ont été le lieu et le moyen de la diffusion d'une masse de connaissances et de savoir-faire. Les techniques de la comptabilité, de la gestion associative, de la conduite des réunions ont souvent joué un rôle positif dans la capacité d'organisation des paysans à l'échelle multi-villageoise. Des règles de fonctionnement qui font de l'égalité une référence suprême, de la souveraineté des membres un principe de base, constituent des jalons importants dans le processus de prise de parole des catégories sociales marginalisées, de la quête de démocratie.

14 Cependant, dans de très nombreux cas, les techniques d'organisation et de fonctionnement n'ont pas été le résultat d'un processus de valorisation de savoirs endogènes. De fait, pour coller aux statuts ou règlements des ONG, tels que le stipule la réglementation officielle, le soin de la rédaction a souvent été confié à des juristes indépendants. Pour la majorité des membres des associations, la connaissance des règlements et de leur signification est généralement hors de portée.

15 2- Les financements accordés par les partenaires ont été les éléments décisifs de la réalisation d'infrastructures, d'œuvres sociales éducatives, d'activités économiques. L'offre de subvention a conduit de nombreuses organisations paysannes à faire de la recherche de fonds pour la réalisation de micro-projets de développement leur objet principal. Ainsi, l'existence de cette offre et la relative facilité d'accéder à des financements entraînent le "refus" d'une recherche des causes durables de la situation vécue. Pourtant, les recherches sur la crise agricole en Afrique indiquent que ce n'est pas une fatalité. Elles mettent en lumière l'inefficience des politiques nationales dans les domaines aussi divers que la Recherche, le système de commercialisation, d'approvisionnement...

16 Cependant, le choix des organisations est compréhensible pour les raisons suivantes. D'abord la recherche et la mise en œuvre d'une nouvelle redistribution des ressources 
nationales ou communales (en vue de la réalisation d'infrastructures) ont un coût élevé ; il en est de même des politiques nationales sectorielles, d'autant plus que les voies de "sortie de crise" n'apparaissent pas de manière évidente. Par ailleurs, la réalisation d'infrastructures et d'œuvres d'éducation sans coût significatif pour les membres (temps de réunion et de mobilisation, conflits, cotisations...) est jugée préférable par les leaders parce que cela contribue à asseoir leur légitimité. Celle-ci est à son tour le moyen de construire des réseaux de clientélisme, de construction d'un pouvoir et d'insertion dans l'arène politique nationale.

17 3- Les coûts de maintenance des infrastructures et de personnel (animateurs, gestionnaires) des associations ont atteint un niveau souvent élevé pour les associations les plus anciennes. De nombreuses voix parmi les partenaires financiers se sont élevées pour demander une réduction progressive de l'engagement financier. Pour ce faire, leaders d'associations et bailleurs de fonds préconisent la mise en place d'entreprises génératrices de revenus, pour les membres mais aussi et surtout pour l'association elle-même.

18 Mais une association peut-elle prendre en charge sur la base d'activités dites rentables le financement d'activités de santé et d'éducation? Il est permis d'en douter. Cela met en lumière des enjeux liés à la politique d'ajustement structurel et de désengagement de l'État. La mise en place d'activités "rentables" ayant peu à voir avec les activités agricoles orientent les associations paysannes dans le sens de la construction de consortiums réunissant des activités disparates dont le but est de prendre en charge les tâches de financement des projets de développement

19 Dans cette perspective, c'est l'identité même des organisations paysannes qui se dilue. On s'éloigne alors des conditions pouvant permettre l'évolution des formes actuelles vers un mouvement social.

\section{AUTEUR}

DANIEL THIÉBA

Dakar 